Ros, G. y Rodríguez Laguna, M.T. (2021). Influencia del aula invertida en la formación científica inicial de Maestros/ as: beneficios en el proceso de enseñanza-aprendizaje, actitudes y expectativas hacia las ciencias. Revista de Investigación Educativa, 39(2), pp-pp.463-482.

DOI: http://dx.doi.org/10.6018/rie.434131

\title{
Influencia del aula invertida en la formación científica inicial de Maestros/as: beneficios en el proceso de enseñanza-aprendizaje, actitudes y expectativas hacia las ciencias
}

\section{Influence of the flipped classroom in the scientific training of pre-service teachers: benefits in the teaching-learning process, attitudes and expectations towards science}

\author{
Germán Ros ${ }^{(*) !}$ y M. Teresa Rodríguez Laguna ${ }^{(* *)}$ \\ Universidad de Alcalá. Alcalá de Henares. Madrid. España. \\ * Departamento de Física y Matemáticas. \\ **Departamento de Química Analítica, Química Física e Ingeniería Química.
}

\begin{abstract}
Resumen
La metodología de aula invertida tiene cada vez mayor difusión en el ámbito universitario pero sus implicaciones no están plenamente analizadas aún. En este trabajo se destacan los aspectos clave para una implementación exitosa del aula invertida y se incluyen objetivos didáctico-disciplinares imprescindibles para el aprendizaje significativo de las Ciencias Experimentales. El estudio se ha realizado en el contexto de la asignatura de Ciencias de la Materia y la Energía en tres titulaciones vinculadas al Grado en Magisterio de Educación Primaria y abarca más de 200 estudiantes, tanto en el grupo experimental como en el grupo control. Este estudio se ha abordado a través de tres instrumentos: el rendimiento académico de las distintas partes de la asignatura; grupos de discusión del personal docente implicado; y cuestionario final cumplimentado por el alumnado. El análisis de los mismos evidencia una mejora significativa en los resultados académicos de forma mayoritaria, al igual que un cambio favorable en sus expectativas como futuros docentes de Ciencias y en el desarrollo de actitudes positivas hacia
\end{abstract}

Correspondencia: Germán Ros Magán, german.ros@uah.es, Facultad de Educación, C/ Madrid 1, 19001, Guadalajara. 
las Ciencias. Además, se analizan los beneficios logrados durante el proceso de enseñanzaaprendizaje, tales como el desarrollo de la capacidad de metacognición, el cambio de roles del personal docente y el alumnado y una mayor retroalimentación y nivel cognitivo alcanzados. Por último, se discuten estos resultados en base a dichos aspectos clave del aula invertida con la inclusión de los objetivos didácticos-disciplinares propios de las Ciencias Experimentales, así como la visión del personal docente a lo largo del mismo.

Palabras clave: métodos de enseñanza; educación superior; logro académico; actitudes de los estudiantes.

\section{Abstract}

The flipped classroom methodology is becoming increasingly widespread in the university environment but its implications have not been fully analyzed yet. This study highlights the key aspects for a successful implementation of the inverted classroom and formulates didactic objectives that are essential for the meaningful learning of Experimental Sciences. The study has been carried out in the context of the subject Sciences of Matter and Energy in three degrees linked to the Degree in Teaching in Primary Education, and covers more than 200 students, both in the experimental group and in the control group. This study has been tackled through three instruments: the academic performance of the different parts of the subject; discussion groups of the teaching staff involved; and the final questionnaire completed by the students. The analysis shows a significant improvement in the academic results in the majority, as well as positive results in their expectations as future science teachers and in the development of positive attitudes towards science. In addition, the benefits achieved during the teaching-learning process are analyzed, such as the development of meta-cognition capacity, the change of roles of teachers and students, a better feedback, and a higher level of cognitive achievement. Finally, these results are discussed on the basis of those key aspects of the inverted classroom with the inclusion of the didactic objectives typical of Experimental Sciences, as well as the view of the teaching staff throughout it.

Keywords: teaching methods; higher education; academic achievement; student attitudes.

\section{Introducción}

La metodología conocida como aula invertida (flipped classroom en inglés) tiene su origen en 2007 cuando Jon Bergmann y Aaron Sams comenzaron a grabar sus presentaciones en video como material adicional para sus estudiantes (Bergmann y Sams, 2012). Esta metodología ha ido asentando sus bases fundamentales en 4 premisas, cuyas iniciales conforman el acrónimo FLIP (Hamdan et al., 2013): flexible environments (ambiente flexible); learning culture (cultura de aprendizaje); intentional content (contenido intencional); y profesional educators (educadores profesionales). En otras palabras, el personal docente debe ser flexible en sus estrategias de enseñanza, cuidar el material para fomentar el estudio previo del alumnado y su reflexión acerca del mismo, así como propiciar un cambio de rol del estudiante hacia un papel más activo y del propio profesorado hacia una labor centrada en el seguimiento y la evaluación del proceso de aprendizaje en su conjunto. Se trata, por tanto, de un modelo que responsabiliza al estudiantado de su propio aprendizaje (Tourón y Santiago, 2015). 
Prieto Martín (2017) indica los aspectos clave para implementar de forma adecuada y exitosa el aula invertida. Estos se recogen en la Figura 1 junto con los objetivos correspondientes.

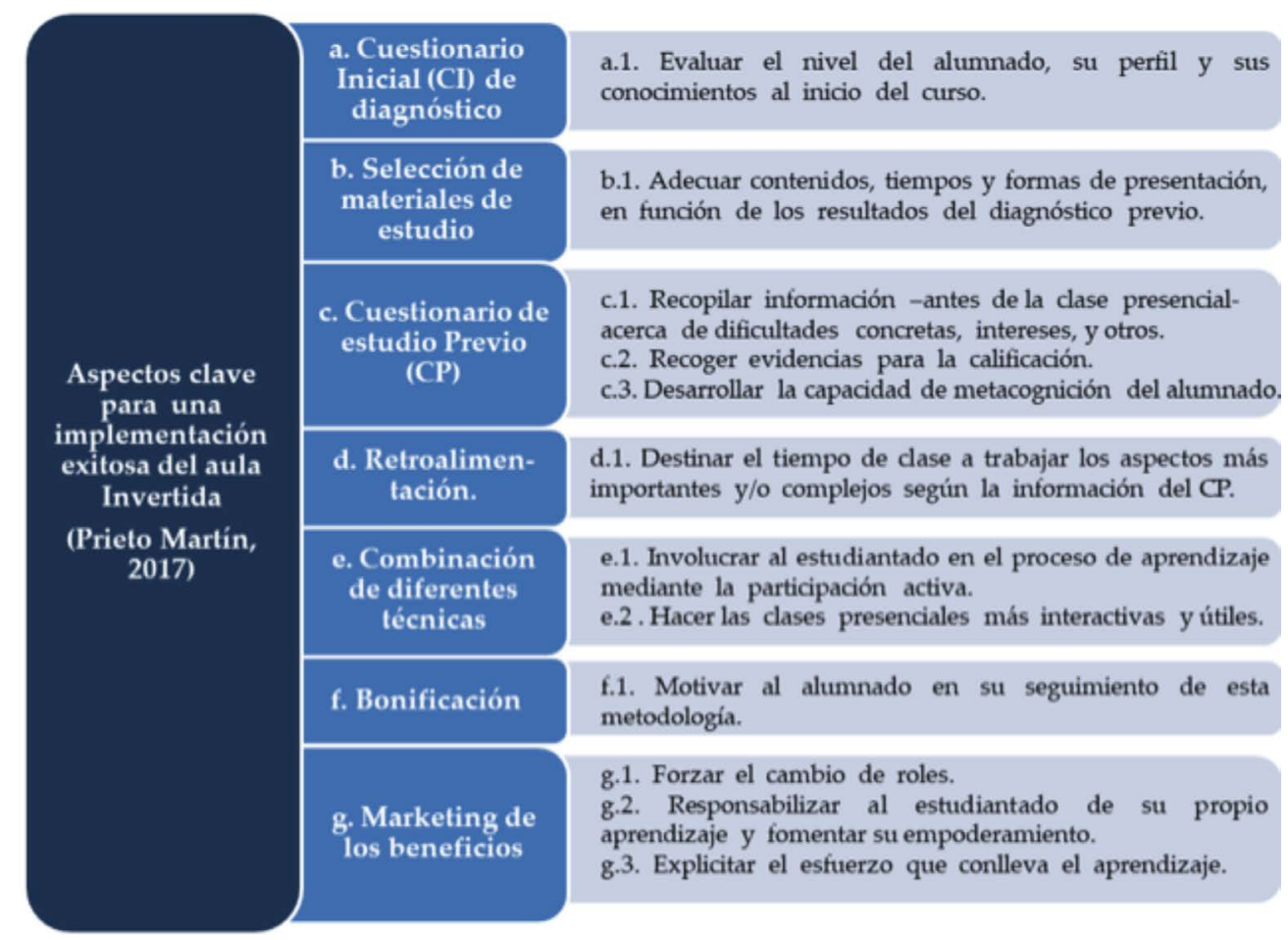

Figura 1. Aula invertida: aspectos clave y objetivos.

En este sentido, Martín y Tourón (2017) citan diferentes estudios en los que se indican algunos factores que son propios del aula invertida bien aplicada:

Algunos de los factores que intervienen en la mejora del aprendizaje se deben a la interacción que se produce entre el profesorado y el alumnado, las actividades llevadas en el aula, el debate, el tiempo para la reflexión de las preguntas . . y la incorporación de diferentes estrategias de enseñanza. (p. 190)

Son diversos los estudios que reportan ventajas derivadas de la implementación del aula invertida. En ellos se resalta que se favorece, mejora o aumenta: la atención a la diversidad (García Barrera, 2013); las estrategias para aprender a aprender, como es la metacognición (Yilmaz y Baydas, 2017); la motivación, autorregulación del aprendizaje y el rendimiento académico (Hinojo Lucena et al., 2019). Todos ellos íntimamente relacionados con los principios motivacionales propuestos por Alonso Tapia (1998), y que forman parte de los aspectos relacionados con, lo que Tourón y Santiago (2015) denominan, la "diferenciación" (adaptación que da respuesta a las singularidades de cada estudiante: capacidades, intereses, etc.). 
No obstante, esta metodología presenta algunos inconvenientes. Respecto del estudiante se destacan dos en la bibliografía: primero, el esfuerzo adicional comparado con la clase tradicional, debido al cambio de roles, al aumento de horas de aprendizaje autónomo, así como la mayor responsabilidad de su propio aprendizaje; segundo, la necesidad de un mínimo de alfabetización digital y de contar con los recursos apropiados (Aguilera-Ruiz et al., 2017; Miller, 2012; Nielsen, 2012). Esta última argumenta, además, que el cambio metodológico no es de por sí suficiente, siendo necesario un cambio educativo y estructural. Por ejemplo, el hecho de adelantar materiales y que el alumnado los lea o visualice no implica aprendizaje y se afirma que esto es una extensión de una pedagogía tradicional. Asimismo, por parte del personal docente se requiere un alto nivel de dedicación para programar el curso de manera más exhaustiva, buscar o crear materiales adecuados, realizar un seguimiento continuado de los cuestionarios y preparar las clases en función de ellos.

Además, en un estudio reciente sobre la influencia de los factores externos en la optimación del aprendizaje mediante aula invertida, se ha constatado que un contexto familiar adecuado seguido de los mecanismos de autorregulación (entre ellos, la autonomía y la motivación) son las variables con mayor incidencia (Mengual Andrés et al., 2020).

En lo que respecta a estudios recientes contextualizados en el Grado en Magisterio en España (González-Gómez et al., 2017; Martín y Tourón, 2017; Salinas Martínez et al., 2018), todos ellos reportan resultados positivos en el grupo experimental respecto del grupo control. Sin embargo, Sola Martínez et al., (2019), concluyen un posible sesgo positivo en las publicaciones que utilizan como criterio el rendimiento académico a partir de un meta-análisis de 12 investigaciones recientes (cinco últimos años) en el nivel universitario. Por ello, dicho grupo de investigación se decanta por dos posibles supuestos: que el aula invertida efectivamente produzca una mejora en el rendimiento académico o que la comunidad investigadora no publique los estudios donde no haya tasa de éxito. Del Arco Bravo et al. (2019) reportan que el cambio de roles no siempre es percibido por el alumnado y demandan una mayor formación del profesorado en el modelo de aula invertida; además confirman -desde la voz del estudiantado- una mayor motivación, pero concluyen que este modelo pedagógico-didáctico exige un elevado nivel de corresponsabilidad y compromiso por ambas partes.

La investigación que se presenta en este estudio está basada en el análisis de la mejora en el rendimiento académico y en la autorregulación en el aprendizaje del estudiante (metacognición y actitudes), en el contexto de enseñanza-aprendizaje de las Ciencias Experimentales (CC.EE. en adelante) mediante la implementación del aula invertida. Para lograrlo se siguieron las pautas de Prieto Martín (2017) (véase la Figura 1), complementando sus objetivos con otros que creemos imprescindibles para el aprendizaje significativo de las CC.EE., tal y como se recoge en la literatura al respecto y que se explicitan en la Figura 2. 


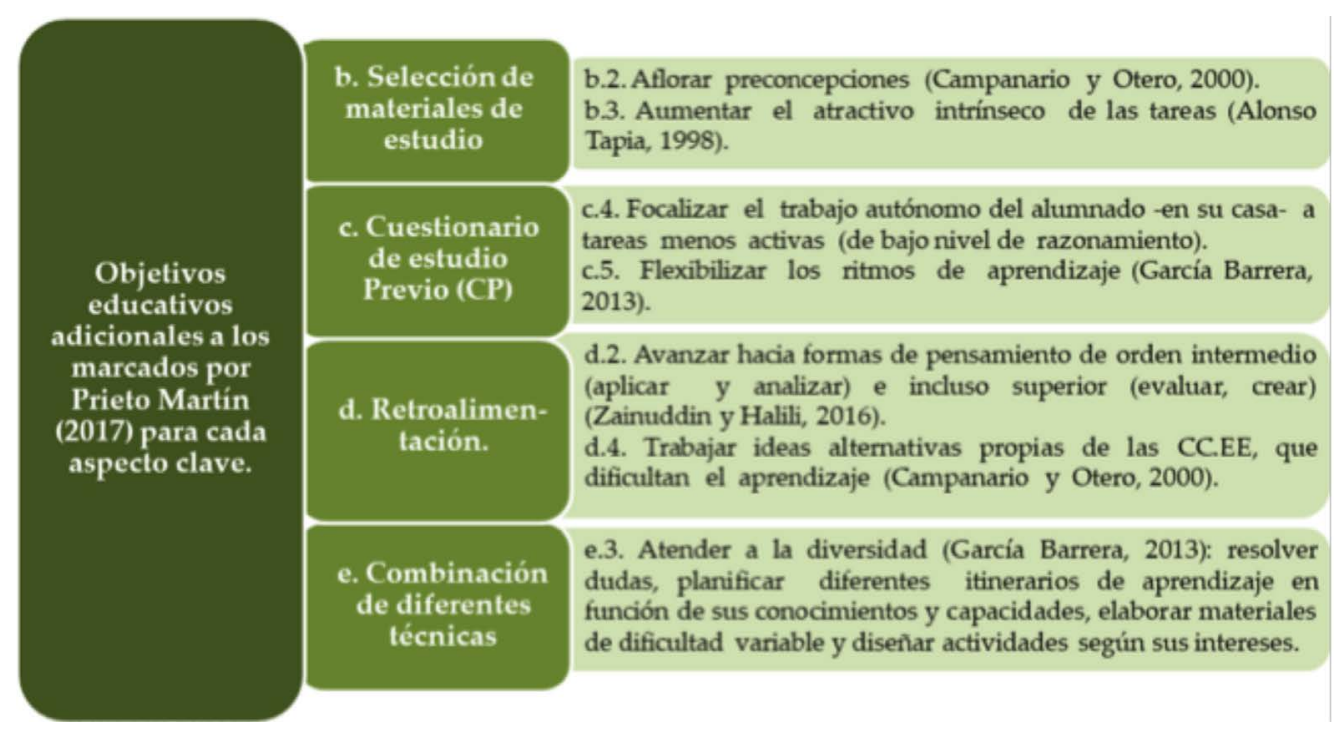

Figura 2. Objetivos educativos adicionales para el aprendizaje significativo de las CC.EE. correspondientes a algunos de los aspectos clave del aula invertida.

Estos objetivos pretenden ofrecer solución al conjunto de causas que, según Campanario y Otero (2000), subyacen en el fracaso del aprendizaje significativo de las CC.EE.: - lo que los alumnos saben (ideas previas), saben hacer (estrategias de razonamiento), creen (concepciones epistemológicas) y creen que saben (metacognición) - (p. 156). Ello exige trabajar de forma activa -con los docentes y con los compañeros y las compañeras- tanto actividades conceptuales que incidan en las dificultades de aprendizaje derivadas de las ideas alternativas (espontáneas, inducidas y analógicas), como procesuales (resolución de problemas y ejercicios, experiencias de aula, experimentación, debates, ) y actitudinales, propias del quehacer científico. En este sentido del Arco Bravo et al. (2019) indican que: - a los docentes les exige conocer los problemas de comprensión y errores conceptuales de los alumnos, para activar mecanismos de apoyo y orientación en el proceso de aprendizaje- (p.466). Igualmente, Tourón y Santiago (2015) señalan 15 aspectos a tener en cuenta que están relacionados con elementos esenciales para la "diferenciación", entre los que se encuentran: el conocimiento de los niveles de partida y la clasificación de errores.

Por otro lado, diversos estudios han mostrado el declive en las actitudes positivas hacia las ciencias entre los 10 y los 15 años (Lupión-Cobos et al., 2019; Vázquez-Alonso y Manassero-Mas, 2015), por lo que es urgente actuar desde la etapa de Educación Primaria. De ahí la relevancia de mejorar las actitudes hacia las ciencias en el alumnado de Magisterio puesto que serán los y las responsables de la formación científica temprana de la futura ciudadanía. Tal y como resaltan Dávila Acedo et al. (2015), en un estudio realizado con Maestros y Maestras en formación inicial, es crucial que estos y estas expresen emociones positivas como la confianza para el desarrollo de actitudes favorables hacia las ciencias y su futura labor. 
Además, según los principios básicos de la investigación desarrollada por profesionales de la educación (Cochran-Smith y Lytle, 2015), el profesorado es personal activo dentro del proceso de enseñanza-aprendizaje, por lo que en este estudio es considerado como elemento indispensable en la evaluación de la innovación metodológica y de sus posibles beneficios.

\section{Método}

\section{Objetivos}

Este trabajo pretende responder las siguientes preguntas de investigación (PI) acerca de la implementación del aula invertida en el contexto de la educación científica en la formación universitaria de Maestros y Maestras de Educación Primaria:

PI1. ¿Mejora el rendimiento académico?

PI2. ¿Influye en la actitud del alumnado hacia las CC.EE. y en sus expectativas futuras como personal docente de Ciencias?

PI3. ¿Qué beneficios produce en el proceso de enseñanza-aprendizaje de las CC.EE.?

Para ello, se han planteado los siguientes objetivos de investigación:

O1. Comparar el rendimiento académico, entre el grupo control (GC) y el grupo experimental (GE), obtenido a partir de las calificaciones de las pruebas parciales, las prácticas de laboratorio y el registro de los cuestionarios previos a la sesión presencial.

O2. Conocer la percepción del alumnado del GE, mediante un cuestionario final, sobre la propia metodología y su repercusión sobre: el desarrollo de habilidades metacognitivas, las actitudes hacia las CC.EE. y las expectativas como futuros docentes de Ciencias.

O3. Recoger la percepción del equipo docente, a través de grupos de discusión, sobre la implementación de la metodología.

O4. Analizar globalmente las consecuencias del aula invertida en: las calificaciones; las actitudes y expectativas hacia las ciencias; la metacognición y otras repercusiones derivadas del propio proceso de implementación, todo ello mediante interrelación de la información recabada con los tres instrumentos: rendimiento académico, grupos de discusión y cuestionario final.

\section{Población y Muestra}

La muestra del estudio está formada por el alumnado de tres titulaciones vinculadas con la formación inicial de Maestros y Maestras en la Universidad de Alcalá: Grado en Magisterio de Educación Primaria (GMEP), subdividido en tres grupos de clase (denominados A, B y C); Doble Grado en Humanidades y en Magisterio de Educación Primaria (DGHP) y Doble Grado en Magisterio de Educación Infantil y en Magisterio de Educación Primaria (DGIP). Las notas de corte varían de una titulación a otra, incluso dentro de GMEP ya que el grupo $\mathrm{B}$ es un grupo bilingüe con otros requisitos de acceso. Por ello, para responder a la primera pregunta de investigación (PI1) se analizará cada grupo por separado. 
El GE, para responder a las preguntas de investigación planteadas, está conformado por el alumnado del curso 2018/19 en las tres titulaciones indicadas. Para analizar la posible mejora en el rendimiento académico (PI1), se elige como GC al alumnado del curso anterior (2017/18) en las titulaciones GMEP y DGHP, ya que el DGIP todavía no se había implantado.

El contexto de este estudio es la asignatura obligatoria de Ciencias de la Materia y la Energía, de 8 créditos ECTS. Consta de dos bloques, uno de Física y otro de Química. La mitad de las sesiones se dedican a clases teóricas y la otra mitad a prácticas de laboratorio. En ambos grupos, control y experimental, la distribución en bloques teórico/prácticos, los contenidos, el personal docente implicado (4 en total, 2 de Física y 2 de Química), los criterios de evaluación y calificación (excepto una bonificación por el seguimiento del aula invertida como se comentará más adelante) y las herramientas de evaluación han sido los mismos. Así pues, las diferencias entre ambos grupos se deben esencialmente en la implementación del aula invertida en el GE comparado con una metodología más tradicional en el GC, esta última basada en la transmisión de conocimiento por parte del docente mediante clase expositiva y deducción de aplicaciones, que el alumnado practica en casa.

El número de estudiantes en cada grupo y titulación se indica en la Tabla 1. La muestra final del estudio es de 218 discentes en el GE y 211 en el GC, así como el equipo de profesorado, conformado 4 docentes.

Tabla 1

Muestra del estudio. Número de estudiantes por titulación y grupo de clase

\begin{tabular}{cccccc}
\hline $\begin{array}{c}\text { Titulación } \\
\text { Grupo }\end{array}$ & GMEP & GMEP & GMEP & $\begin{array}{c}\text { DGHP } \\
\text { único }\end{array}$ & $\begin{array}{c}\text { DGIP } \\
\text { único }\end{array}$ \\
\hline Grupo Control (GC) & 93 & 26 & 54 & 38 & --- \\
Grupo Experimental (GE) & 60 & 34 & 54 & 27 & 43 \\
\hline
\end{tabular}

En cuanto al nivel de conocimientos científicos (objetivo a.1, Figura 1) la puntuación media en el cuestionario inicial fue de 27 (sobre 100) con una desviación estándar de 15 puntos. El grupo C obtuvo peores resultados pero no con una diferencia significativa.

\section{Instrumento}

Los instrumentos utilizados han sido principalmente tres: la medición del rendimiento académico, un cuestionario final y grupos de discusión entre el profesorado de la asignatura, tal como se muestra en la Figura 3. 


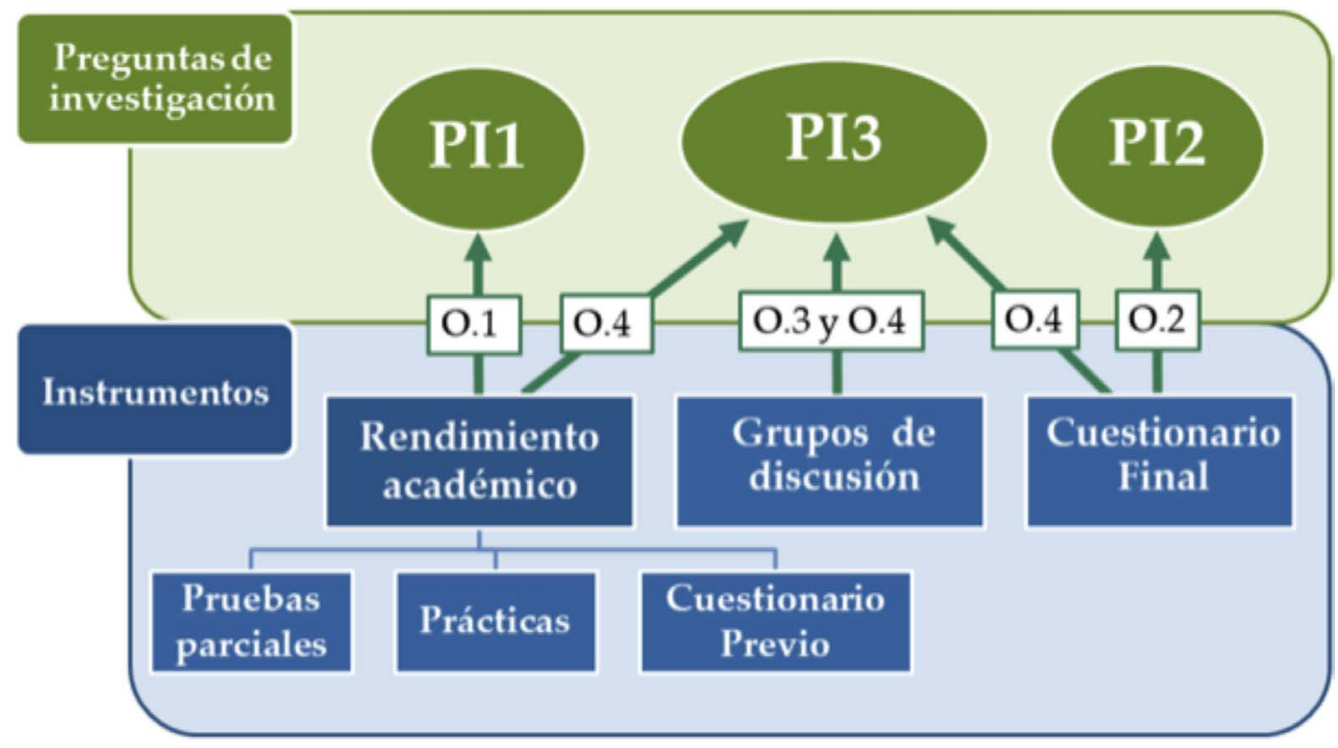

Figura 3. Relación entre los distintos instrumentos, los objetivos de investigación y sus correspondientes preguntas.

Para medir el rendimiento académico, como se adelanta en la Figura 3, se han considerado las calificaciones recogidas a través de tres herramientas:

- Pruebas parciales escritas (40\% de la calificación) con preguntas breves contextualizadas en la vida cotidiana y orientadas a medir la comprensión; así como ejercicios y problemas de aplicación.

- Sesiones prácticas (50\% de la calificación): tanto de calificaciones grupales, que se evaluaron mediante informes o rúbricas, como individuales, evaluadas con cuestionarios sincrónicos con la herramienta web Socrative; y también se tuvieron en cuenta otros aspectos, tales como, la colaboración, el cumplimento de las normas de seguridad e higiene, etc.

- Registro de los cuestionarios previos: supone una bonificación en la calificación $(10 \%)$ que se calcula de modo proporcional al número de cuestionarios previos respondidos de manera razonada. Asimismo, su seguimiento permite conocer el nivel de implicación y esfuerzo continuado de los estudiantes. Se ha utilizado el cuestionario previo propuesto por Prieto Martín (2017, pp. 148-149).

En lo que respecta a la bonificación, por ser un elemento del aula invertida, no es de aplicación en el GC y en consecuencia las calificaciones de las sesiones prácticas suponían el $60 \%$ de la calificación. Este hecho se ha tenido en cuenta -como veremos posteriormente- en el análisis de los datos.

Por otro lado, a través de un cuestionario final, se han recogido las evidencias acerca de los beneficios del aula invertida en el proceso de enseñanza-aprendizaje, así como su posible influencia en la actitud de los alumnos y las alumnas hacia las CC.EE. y 
en sus expectativas futuras como Maestros y Maestras de Educación Primaria. Dicho cuestionario final se ha elaborado con respuestas de valoración en escala Likert de 0 (mínimo) a 5 (máximo), con un número par de posibilidades para evitar la tendencia al valor medio (Bisquerra y Pérez-Escoda, 2015). Las afirmaciones relativas a los procesos de metacognición, actitudes y expectativas se seleccionaron de dos cuestionarios muy utilizados y validados en la literatura. Se trata, por un lado, del Motivated Strategies for Learning Questionnaire Manual (García Duncan y McKeachie, 2005), que es un test muy amplio que mide diversos aspectos relacionados con la autorregulación del estudiante; por otro, el Cuestionario R-SPQ-2F (Biggs et al., 2001) más orientado hacia el enfoque de estudio del alumnado. El cuestionario utilizado se muestra por categorías (metodología, metacognición, actitudes y expectativas) en la Tabla 2. Cabe indicar que las afirmaciones del primer bloque (MI1 a MI8 de la Tabla 2) se realizan para Física y Química por separado a fin de evaluar y considerar posibles diferencias entre el personal docente.

Se ha realizado un test Alpha de Cronbach $(\alpha)$ con el objetivo de comprobar la fiabilidad y consistencia interna del cuestionario (Loewenthal y Lewis, 2018). Los resultados del test han sido positivos en todas las categorías y los valores obtenidos se muestran en la Tabla 2.

Adicionalmente -en el cuestionario final- se solicitó al alumnado que indicara 5 palabras que reflejaran su opinión, actitud o sentimiento acerca de la metodología de aula invertida.

Por último, se realizaron grupos de discusión entre el personal docente y se recogieron anotaciones en cuadernos de campo que fueron registrados y analizados de forma independiente por cada investigador/a y puestos posteriormente en común.

Tabla 2

Afirmaciones del cuestionario final de cada categoría analizada

\section{METODOLOGÍA DE AULA INVERTIDA $(\alpha=.84)$}

MI.1 Las clases de teoría han sido mejor aprovechadas al centrarse en las dificultades principales de los alumnos y las alumnas.

MI.2 En las clases o fuera de ellas ha existido un diálogo/debate entre estudiantes y/o entre el alumnado y el profesorado mejor que en otras asignaturas.

MI.3 La metodología seguida en general me ha ayudado a comprender mejor las Ciencias de la Materia y la Energía.

MI.4 Comparado con otras asignaturas, estas clases han sido más interactivas.

MI.5 La metodología ha fomentado una relación positiva entre alumnado y profesorado.

MI.6 El avanzar de forma progresiva y continuada a lo largo de la asignatura me proporcionaba cierta sensación de éxito y satisfacción.

MI.7 Esta metodología me ha hecho dedicar más horas de estudio continuado que en otras asignaturas.

MI.8 Me gustaría que otras asignaturas utilizasen un esquema invertido similar al seguido en esta asignatura. 


\section{METACOGNICIÓN ( $\alpha=$.72)}

MC.1 Habitualmente me pregunto sobre lo que leo y reflexiono sobre ello.

MC.2 Incluso cuando no lo entiendo bien intento por mí mismo/a continuar.

MC.3 Reflexiono acerca de mis dudas y lo que comprendo y no comprendo.

MC.4 Intento comprender las ideas y no aprenderlas de forma memorística.

MC.5 Necesito comprender los conceptos y no me basta con memorizar para aprobar los exámenes.

MC.6 Me autoevalúo en temas importantes hasta que los entiendo por completo.

\section{ACTITUDES $(\alpha=.78)$}

A.1 Me gusta mucho aprender sobre ciencias.

A.2 Mi percepción sobre las ciencias ha mejorado mucho tras esta asignatura.

A.3 El estudio de las ciencias en la escuela es muy relevante.

A.4 Había temas o ejemplos que se relacionaban fácilmente con aplicaciones de la vida cotidiana.

A.5 Había temas que podía relacionar con mis conocimientos previos ya que los había estudiado antes o visto en algún momento.

A.6 Me he divertido en este curso.

\section{EXPECTATIVAS FUTURAS $(\alpha=.87)$}

E.1 Tras esta asignatura mi propia percepción sobre mis conocimientos científicos ha mejorado significativamente.

E.2 Tras esta asignatura me siento preparado/a o más preparado/a para enseñar ciencias en Educación Primaria.

E.3 Me siento capaz de aprender -por mí mismo/a, en el futuro- sobre más conceptos científicos.

Nota. Alpha de Cronbach $(\alpha)$ de cada categoría

\section{Procedimiento de recogida y análisis de datos}

Los datos para medir el rendimiento académico se han recogido a lo largo del cuatrimestre. Concretamente, se han realizado 4 pruebas parciales, 14 sesiones prácticas y los cuestionarios previos antes de cada clase presencial mediante un cuestionario online de Google Form asincrónico. Por otro lado, el cuestionario final se realizó el último día a través de la herramienta web con los dispositivos móviles de los estudiantes. Respecto a los grupos de discusión del profesorado, se llevaron a cabo al inicio para programar todo el curso, así como durante y a la finalización de las clases para valorar el proceso y el resultado de la aplicación del aula invertida.

Los parámetros y análisis estadísticos de los datos recabados que se han realizado han sido los siguientes: 
Análisis de normalidad de las distribuciones de las calificaciones. Se ha utilizado el test de Shapiro-Wilk. Se comprueba que no se cumple la hipótesis de normalidad en ningún caso.

- Homocedasticidad (homogeneidad de varianzas) de las distribuciones de las calificaciones a comparar entre el GE y el GC. Se ha realizado mediante el test F de Fisher. Se comprueba que, en varios casos, no se cumple la homocedasticidad.

- Análisis de la significación de las diferencias en el resultado académico entre el GE y el GC. Ante los resultados de los test anteriores, se ha elegido el test de significación estadística de la U de Mann-Whitney, obteniéndose el p-valor y la diferencia entre ambos grupos.

- Parámetros estadísticos descriptivos de las respuestas al cuestionario final.

\section{Resultados}

\section{Efecto del aula invertida sobre el rendimiento académico}

La Figura 4 muestra los resultados escalados sobre 10 puntos tanto para la parte de exámenes como las sesiones prácticas para los bloques de Física y Química. La bonificación solo se incluye en la calificación final. Se ha escogido un diagrama de cajas para que se aprecie la dispersión y asimetría de las distribuciones.

Los resultados del test $U$ de Mann-Whitney se muestran en la Tabla 3, donde se indica la diferencia entre el GE y el GC, y si esta es significativa al 95\% de confianza.
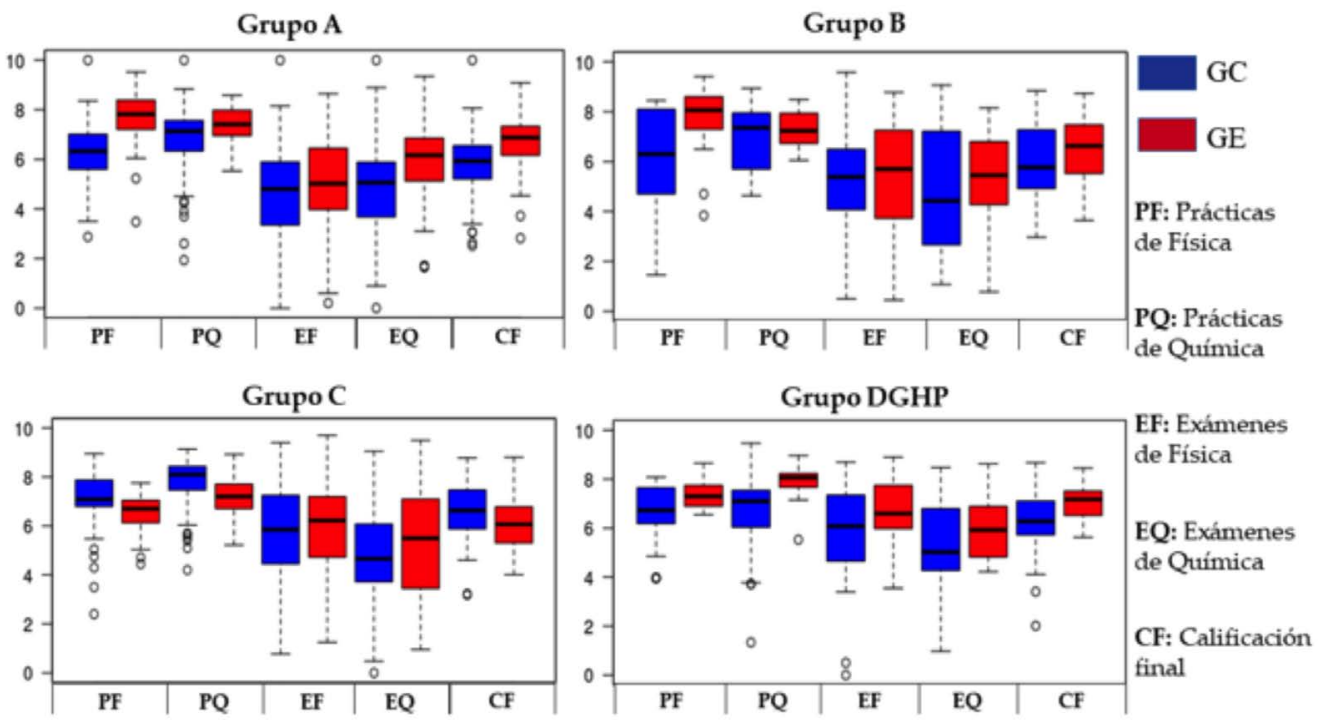

Figura 4. Comparación de las calificaciones obtenidas entre el GC y el GE. 
Tabla 3

Diferencia en el rendimiento académico entre el GE y el GC

\begin{tabular}{lcccc}
\hline \multicolumn{1}{c}{ Partes asignatura } & $\begin{array}{c}\text { GMEP } \\
\text { A }\end{array}$ & $\begin{array}{c}\text { GMEP } \\
\text { B }\end{array}$ & $\begin{array}{c}\text { GMEP } \\
\text { C }\end{array}$ & $\begin{array}{c}\text { DGHP } \\
\text { único }\end{array}$ \\
\hline Prácticas de Física (PF) & $+1.42^{*}$ & $+1.40^{*}$ & $-.52^{*}$ & $+.54^{*}$ \\
Prácticas de Química (PQ) & $+.39^{*}$ & $+.28^{*}$ & $-.77^{*}$ & $+.99^{*}$ \\
Exámenes de Física (EF) & +.35 & +.31 & +.25 & +.55 \\
Exámenes de Química (EQ) & $+1.12^{*}$ & +.84 & +.66 & +.50 \\
Calificación Total & $+.89^{*}$ & +.55 & $-.51^{*}$ & $+.63^{*}$ \\
\hline
\end{tabular}

Nota. ${ }^{*} \mathrm{p}<.05$

De estos resultados del rendimiento académico se pueden destacar varios aspectos:

- En tres de los cuatro grupos la implantación del aula invertida ha mejorado el rendimiento académico, siendo significativa en dos de ellos (A y DGHP).

- De forma llamativa en el grupo C existe un resultado negativo. Se debe esencialmente a haber obtenido calificaciones más bajas en las prácticas (Tabla 3) que representan el 50\% de la calificación total. Buscando las causas de la diferencia de este grupo con el resto, encontramos dos: está formado por un alumnado con menor bagaje científico previo, ya que en este grupo el porcentaje de estudiantes que no proceden de Bachillerato Científico ni de Ciencias Sociales es mucho más alto que en el resto (64\% frente a $21-43 \%$ del resto); además, es el grupo con menor seguimiento y cumplimentación de los cuestionarios previos (solo un $25 \%$ de estudiantes ha contestado por encima del $50 \%$ de los mismos), hecho este último clave en el rendimiento académico como se discute más adelante.

- En los exámenes, a excepción de las pruebas de Química del grupo A, no se ha encontrado una diferencia significativa entre los resultados del GE respecto al GC.

- En las prácticas la mejora en las calificaciones es alta y significativa en todos los grupos menos en el grupo $\mathrm{C}$ por las causas antes mencionadas.

\section{Influencia en las actitudes y expectativas hacia las ciencias}

La Tabla 4 muestra las respuestas del cuestionario final (Tabla 2) de las categorías de actitudes y expectativas, respectivamente. Los datos se presentan sin disgregar por grupos de análisis ni bloques de la asignatura, puesto que no se han encontrado diferencias significativas entre ellos. 
Tabla 4

Porcentaje de respuesta para cada opción Likert para las categorías: actitudes y expectativas

\begin{tabular}{lcccccc}
\hline & $\mathbf{0}$ & $\mathbf{1}$ & $\mathbf{2}$ & $\mathbf{3}$ & $\mathbf{4}$ & $\mathbf{5}$ \\
\hline A.1 & 1.0 & 4.9 & 11.8 & 29.4 & 27.9 & 25.0 \\
A.2 & .5 & 1.5 & 9.8 & 23.5 & 33.3 & 31.4 \\
A.3 & .5 & .5 & 3.4 & 13.2 & 32.9 & 49.5 \\
A.4 & 0 & 0 & 1.0 & 9.3 & 23.0 & 66.7 \\
A.5 & 4.9 & 6.9 & 13.2 & 19.1 & 25.0 & 30.9 \\
A.6 & 3.9 & 1.5 & 8.8 & 23.5 & 30.9 & 31.4 \\
\hline E.1 & .5 & 1.5 & 6.8 & 19.1 & 41.7 & 30.4 \\
E.2 & .5 & 2.9 & 9.3 & 21.6 & 29.9 & 35.8 \\
E.3 & 2.5 & 1.5 & 5.4 & 23.0 & 35.3 & 32.3 \\
\hline
\end{tabular}

Nota. Los códigos corresponden a las afirmaciones de la Tabla 2

Como puede verse en los resultados del cuestionario final (Tabla 4), todas las preguntas referentes a actitudes hacia las Ciencias tienen una alta puntuación (máximos en 4 o 5, excepto una pregunta con máximo en 3). La percepción de las CC.EE. mejora mucho tras la aplicación del aula invertida (A2) gracias a las estrategias de andamiaje utilizadas (contextualización con ejemplos de la vida cotidiana y conexión con sus conocimientos previos, preguntas A4 y A5), resultando una actitud positiva hacia el estudio de las CC.EE. (A1, A6).

Además, la gran mayoría del alumnado siente que sus conocimientos han mejorado (E1) y más importante aún, se perciben más capaces de enseñar Ciencias (E2) y de aprender de manera autónoma (E3).

\section{Beneficios del aula invertida}

La Tabla 5 muestra las respuestas al cuestionario final referentes a la evaluación de la propia metodología y del desarrollo de habilidades metacognitivas del alumnado. $\mathrm{Al}$ igual que en la Tabla 4, los datos se presentan sin disgregar por las mismas razones.

Tabla 5

Porcentaje de respuesta para cada opción Likert de las categorías: metodología de aula invertida y metacognición

\begin{tabular}{lrrrrrc}
\hline & $\mathbf{0}$ & $\mathbf{1}$ & $\mathbf{2}$ & $\mathbf{3}$ & $\mathbf{4}$ & $\mathbf{5}$ \\
\hline MI.1 & 4.4 & 5.6 & 12.0 & 19.9 & 31.4 & 26.7 \\
MI.2 & 6.4 & 4.2 & 13.5 & 27.0 & 3.1 & 18.9 \\
MI.3 & 4.9 & 7.6 & 13.7 & 21.1 & 28.2 & 24.5 \\
MI.4 & 2.9 & 3.7 & 9.8 & 16.2 & 3.1 & 37.3 \\
\hline
\end{tabular}




\begin{tabular}{lcccccc}
\hline & $\mathbf{0}$ & $\mathbf{1}$ & $\mathbf{2}$ & $\mathbf{3}$ & $\mathbf{4}$ & $\mathbf{5}$ \\
\hline MI.5 & 2.4 & 2.9 & 9.1 & 14.0 & 31.9 & 39.7 \\
MI.6 & 2.7 & 3.9 & 15.0 & 25.2 & 29.2 & 24.0 \\
MI.7 & 2.2 & 3.7 & 13.0 & 15.7 & 27.2 & 38.2 \\
MI.8 & 8.3 & 1.8 & 9.8 & 29.4 & 27.0 & 14.7 \\
\hline MC.1 & 0 & 1.0 & 1.3 & 26.0 & 37.7 & 25.0 \\
MC.2 & .5 & 0 & 7.9 & 18.1 & 39.2 & 34.3 \\
MC.3 & .5 & 1.0 & 2.9 & 22.1 & 35.8 & 37.7 \\
MC.4 & .5 & 1.5 & 2.0 & 13.7 & 31.8 & 5.5 \\
MC.5 & 1.5 & 1.5 & 2.9 & 17.7 & 22.5 & 53.9 \\
MC.6 & 4.4 & 2.0 & 13.2 & 27.9 & 3.4 & 22.1 \\
\hline
\end{tabular}

Nota. Los códigos corresponden a las afirmaciones de la Tabla 2

A la vista de los resultados del cuestionario final (Tabla 5) se puede afirmar que el estudiantado considera que el aula invertida ha propiciado:

- Una mejora en el nivel de compresión conceptual (MI3) al haber un mayor aprovechamiento de las clases centradas en sus dificultades (MI1). Se logra así un mayor y mejor nivel de retroalimentación lo que se refleja en las calificaciones de los exámenes como se ha analizado antes.

- Un mayor nivel de estudio comparado con metodologías tradicionales (MI7), lo que unido a su buen resultado académico se traduce, en general, en una sensación de éxito y satisfacción a lo largo del curso (MI6).

- El logro en el cambio de roles. Afirman que ha habido una mejor relación y mayor diálogo, tanto con el profesorado y como entre ellos mismos (MI2, MI5), haciendo la clase más interactiva (MI4).

- Un mayor desarrollo metacognitivo. El alumnado ha entendido la necesidad de reflexionar al abordar los conceptos con ayuda de los materiales previos (MC1, MC2, MC3), procurando aprender de forma razonada (MC4, MC5). Además, el estudiantado afirma que se han ido autoevaluando durante el proceso (MC6), aspecto que enfatiza el aula invertida al obligar al alumno a expresar sus dudas y certezas en los cuestionarios previos y tratarlas en las clases de forma específica.

Asimismo, a modo valoración global del alumnado (opinión, actitud o sentimiento acerca del aula invertida), entre las 5 palabras recogidas en el cuestionario final destacan en frecuencia los vocablos muy positivos (útil, interactiva, innovadora, motivadora, dinámica, aprendizaje, eficaz, práctica, divertida, participativa, etc.), otras que manifiestan que efectivamente ha provocado una mayor dedicación por su parte (esfuerzo, constancia) y en menor medida palabras negativas (inadecuada, agobiante), que fueron señaladas por estudiantes con menor seguimiento continuo y un rol más pasivo.

Por otro lado, como se ha comentado, un beneficio fundamental del aula invertida es el esfuerzo continuado que esta metodología demanda a los estudiantes. Para evaluar esta continuidad se ha hecho un seguimiento de los cuestionarios previos sin contabilizar la bonificación por responderlos para observar correctamente el efecto del estudio previo en el rendimiento académico. Por ello, en la Figura 5 se muestra la 
calificación final (sobre 9). Como se aprecia existe una correlación positiva significativa entre la calificación y el seguimiento del alumnado.

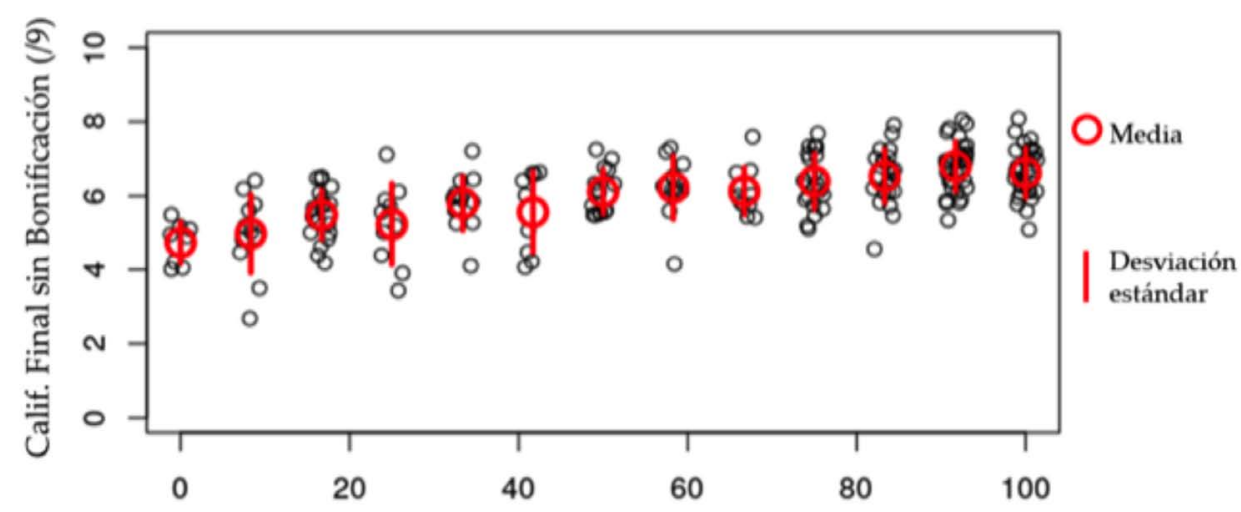

Porcentaje de cuestionarios respondidos

Figura 5. Relación entre la calificación final y el seguimiento de la metodología.

En cuanto a los beneficios para el personal docente, que fueron recogidos en los grupos de discusión, se pueden extraer los siguientes aspectos:

- Amplia retroalimentación para el profesorado a través de la información recibida en los cuestionarios previos, que le permite realizar un seguimiento, adaptar sus clases y materiales, así como trabajar los principios $3^{\mathbf{0}}-6^{\mathbf{0}}$ motivacionales de Alonso Tapia (1998) en las sesiones presenciales.

- Mayor satisfacción en el equipo docente al lograr una mejora significativa de la actitud en clase, en el nivel de participación del alumnado -fruto del estudio previo y de su mayor interés en resolver sus dudas- y en el mayor nivel conceptual alcanzado.

- Se logra la existencia de una relación más fluida y bidireccional que en las clases habituales, por tanto, desde la perspectiva del personal docente también se logra el cambio de roles;

- Se destaca, no obstante, que la carga de trabajo para el profesorado es mucho más alta, sobre todo en la selección de materiales, la revisión de los cuestionarios previos antes de cada clase, la preparación de nuevos materiales que respondan las dudas, dificultades e intereses, etc.

\section{Discusión y conclusiones}

Se ha abordado el análisis de la implementación del aula invertida en el proceso de enseñanza-aprendizaje en la asignatura de Ciencias de la Materia y la Energía (formada por contenidos de Física y Química) en el contexto de tres titulaciones vinculadas la formación inicial de Maestros y Maestras de Educación Primaria. 
Dicha implementación se ha realizado de manera exitosa siguiendo algunos aspectos clave (Martín y Tourón, 2017; Prieto Martín, 2017) con los siguientes objetivos didácticos:

- explicitar los beneficios del esfuerzo continuado que la metodología demanda al alumnado;

- conocer la situación de partida a través de un cuestionario diagnóstico inicial;

- utilizar materiales óptimos, preparando y seleccionándolos considerando el nivel, el tiempo que requieren al estudiante y el soporte. Además, deben ser atractivos para despertar su curiosidad y mantener su atención y constancia (principio $1^{\circ}$, Alonso Tapia, 1998);

- recoger información del alumnado tras el estudio mediante cuestionarios previos a las clases;

- centrar las clases presenciales teóricas en la resolución de dudas y tareas de mayor complejidad;

- otorgar una bonificación al alumno por el seguimiento de la metodología.

A estos se han añadido otros objetivos adicionales enfocados al aprendizaje significativo de las CC.EE., tales como aflorar preconcepciones (Campanario y Otero, 2000) y fomentar formas de pensamiento de orden superior (Zainuddin y Halili, 2016), entre otros (Figura 2).

Se ha analizado el efecto de la clase invertida bajo estos objetivos didácticos-disciplinares en cuatro aspectos: el rendimiento académico del alumnado; sus actitudes hacia las CC.EE.; las expectativas hacia su enseñanza; y, por último, los beneficios tanto para el alumnado como para el profesorado. La Figura 6 recoge las principales conclusiones del estudio como respuesta a las preguntas de investigación planteadas y que son discutidas a continuación.

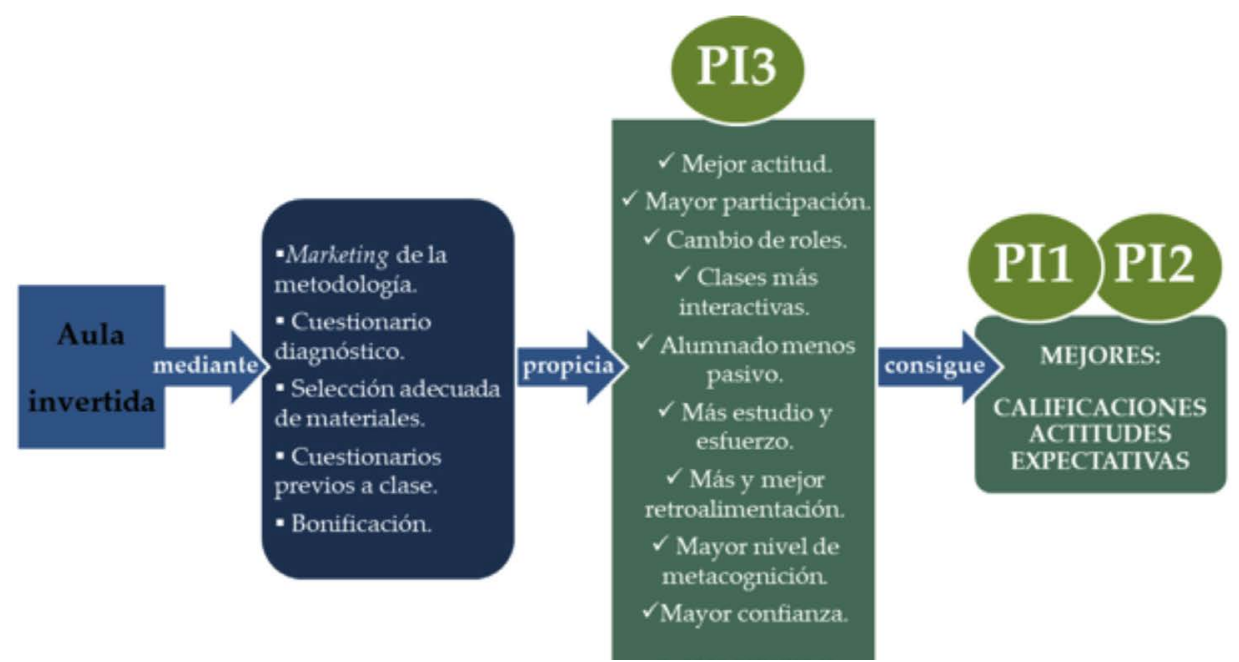

Figura 6. Resumen de las conclusiones del trabajo y respuestas a las preguntas de investigación (PI). 
En cuanto a la influencia de la clase invertida en el rendimiento académico, la valoración global es positiva, con resultados de mejora global respecto al grupo control en la mayoría de grupos analizados. Esta mejora ocurre en las prácticas de forma significativa, excepto en un grupo, y en los exámenes, si bien, en este último caso, aunque la mejora es clara en todos los grupos no resulta estadísticamente significativa. Los resultados negativos en las prácticas de uno de los grupos se muestran en respuesta a la demanda de Sola Martínez et al. (2019). Los resultados están en acuerdo con diversas investigaciones que afirman que el estudio previo propio del aula invertida ayuda a la adquisición y comprensión de los conceptos (Arshad y Imran, 2013). En esa línea González-Gómez et al. (2017), que realizaron aula invertida en esta misma asignatura en la Universidad de Extremadura, encontraron mejoras significativas en los exámenes, pero no en las prácticas.

Por otro lado, nuestros resultados avalan que la aplicación del aula invertida sirve para formar Maestros y Maestras con mayor autoconfianza hacia la enseñanza de las Ciencias y mayor sentimiento de autonomía. Afrontar las Ciencias con confianza y haber logrado desarrollar su capacidad de auto-aprendizaje serán herramientas muy valiosas en su futuro ejercicio profesional. Este resultado se alinea con el estudio de del Arco Bravo et at. (2019) que, a partir de cuestionarios ad-hoc validados, demuestran un cambio de implicación en sus desempeños y un aumento de seguridad en los mismos.

Analizando la opinión del alumnado, se encuentra que la clase invertida propicia beneficios que van desde una mayor comprensión de los conceptos científicos hasta un mayor grado de metacognición durante el proceso, logrando un cambio de roles que ha fomentado mayor estudio por su parte, mayor interactividad y utilidad de las clases presenciales promoviendo una mayor sensación de satisfacción y motivación. Este cambio de roles es destacado por los creadores del aula invertida como lo más relevante de la metodología (Bergmann y Sams 2012).

Estos beneficios están en consonancia con la exigencia reportada por del Arco Bravo et al. (2019), en cuanto al conocimiento didáctico-disciplinar del profesorado, y que en este estudio se evidencian a través de la incorporación de unos objetivos adicionales específicos para las CC.EE., comentados anteriormente, a los pautados por Prieto Martín (2017) para el aula invertida.

Por tanto, se logra una mayor motivación en el alumnado lo que, de acuerdo a los principios motivacionales $2^{\underline{o}}$ y $5^{\circ}$ (Alonso Tapia, 1998), propicia una mayor conciencia del proceso de aprendizaje, un mayor grado de implicación y autonomía en el alumnado y se mejora la confianza antes de asistir a clase (empoderamiento).

Más aún, el estudiantado afirma desear más clases con esta metodología (pregunta MI8) pero con menor rotundidad que en otras preguntas. Esto parece en consonancia con la resistencia del alumnado a asumir un mayor nivel de exigencia y un rol más autónomo y responsable en su aprendizaje (Aguilera-Ruiz et al., 2017; Miller, 2012; Nielsen, 2012).

Profundizando en la relación entre el esfuerzo que exige la metodología y el beneficio en el rendimiento académico, el seguimiento de los cuestionarios previos arroja una situación muy variada. Por ejemplo, un $25 \%$ del alumnado hizo menos del $30 \%$ de ellos, si bien más del 50\% cumplimentaron más del 50\% de los mismos. Este resultado está en consonancia con la postura de Miller (2012), quien señala que el aula invertida crea la oportunidad pero que de por sí no siempre va a conseguir que el alumnado se 
implique en ella. Asimismo, como puede verse en la Figura 5, existe una clara correspondencia entre el nivel de seguimiento del alumnado y el rendimiento académico. Esto pone de manifiesto la relevancia de explicitar los beneficios de esta metodología, desde el primer día y de manera continuada, y del mayor esfuerzo que el alumnado ha de hacer, tal y como propone Prieto Martín (2017).

Por último, el equipo docente también expresa su satisfacción al percibir una mejora en el nivel conceptual alcanzado por el alumnado, el aumento de la participación activa en clase y el logro de un mayor nivel de motivación en los estudiantes. Todo ello, a pesar de la mayor carga de trabajo que supone, especialmente el primer curso que se implementa esta metodología.

\section{Agradecimientos}

A Juan Miguel Campanario y Ana Belén García Varela, docentes de la Universidad de Alcalá, por sus sugerencias en la redacción y organización del artículo.

\section{Referencias}

Aguilera-Ruiz, C., Manzano-León, A., Martínez-Moreno, I., Lozano-Segura, M.C. y Casiano Yanicelli, C. (2017). El modelo flipped classroom. International Journal of Developmental and Educational Psychology, 4(1), 261-266. https://doi.org/10.17060/ ijodaep.2017.n1.v4.1055

Alonso Tapia, J. (1998). Motivación y aprendizaje en el aula: cómo enseñar a pensar. Santillana.

Arshad, K. y Imran, M. A. (2013). Increasing the interaction time in a lecture by integrating flipped classroom and just-in-time teaching concepts. Compass: Journal of Learning and Teaching, 4(7), 1-13. https://doi.org/10.21100/compass.v4i7.84

Bergmann, J. y Sams, A. (2012). Flip your classroom: Reach every student in every class every day. International society for technology in education.

Biggs, J., Kember, D. y Leung, D. Y. (2001). The revised two-factor study process questionnaire: R-SPQ-2F. British journal of educational psychology, 71(1), 133-149. https:// doi.org/10.1348/000709901158433

Bisquerra, R. y Pérez-Escoda, N. (2015). ¿Pueden las escalas Likert aumentar en sensibilidad? REIRE. Revista d'Innovació i Recerca en Educació, 8(2), 129-147. https://doi. org/10.1344/reire2015.8.2828

Campanario, J. M. y Otero, J. (2000). Más allá de las ideas previas como dificultades de aprendizaje: las pautas de pensamiento, las concepciones epistemológicas y las estrategias metacognitivas de los alumnos de Ciencias. Enseñanza de las Ciencias: revista de investigación y experiencias didácticas, 18(2), 155-169.

Cochran-Smith, M. y Lytle, S. L. (2015). Inquiry as stance: Practitioner research for the next generation. Teachers College Press.

Dávila Acedo, M. A., Borrachero Cortés, A. B., Martínez Borreguero, M. G. y Sánchez Martín, J. (2015). Evolución de las emociones que experimentan los estudiantes del grado de maestro en educación primaria, en didáctica de la materia y la energía. 
Revista Eureka sobre Enseñanza y Divulgación de las Ciencias, 12(3), 550-564. https:// doi.org/10498/17609

del Arco Bravo, I., Flores Alarcia, O. y Silva García, P. (2019). El desarrollo del modelo flipped classroom en la universidad: impacto de su implementación desde la voz del estudiantado. Revista de Investigación Educativa, 37(2), 451-469. https:/doi. org/10.6018/rie.37.1.327831

García Barrera, A. (2013). El aula inversa: cambiando la respuesta a las necesidades de los estudiantes. Avances en supervisión educativa, (19), 1-8. https://doi.org/10.23824/ ase.v0i19.118

García Duncan, T. y McKeachie, W. J. (2005). The making of the motivated strategies for learning questionnaire. Educational psychologist, 40(2), 117-128. https://doi. org/10.1207/s15326985ep4002_6

González-Gómez, D., Jeong, J. S., Cañada-Cañada, F. y Gallego Picó, A. (2017). La enseñanza de contenidos científicos a través de un modelo "Flipped": Propuesta de instrucción para estudiantes del Grado de Educación Primaria. Enseñanza de las Ciencias: revista de investigación y experiencias didácticas, 35(2), 71-87. https://doi. org/10.5565/rev/ensciencias.2233

Hamdan, N., McKnight, P. E., McKnight, K. y Arfstrom, K.M. (2013). The flipped learning model: A white paper based on the literature review titled a review of flipped learning. Flipped Learning Network. 3-20. http://researchnetwork.pearson.com/wpcontent/ uploads/WhitePaper_FlippedLearning.pdf

Hinojo Lucena, F. J., Aznar Díaz, I., Romero Rodríguez, J. M., y Marín Marín, J. A. (2019). Influencia del aula invertida en el rendimiento académico. Una revisión sistemática. Campus Virtuales, 8(1), 9-18. http://uajournals.com/ojs/index.php/campusvirtuales/article/view/384

Loewenthal, K. M. y Lewis, C. A. (2018). An introduction to psychological tests and scales. Psychology press. https://doi.org/10.4324/9781315782980

Lupión-Cobos, T. L., Franco-Mariscal, A. J. y Girón Gambero, J. R. (2019). Predictores de vocación en Ciencia y Tecnología en jóvenes: Estudio de casos sobre percepciones de alumnado de secundaria y la influencia de participar en experiencias educativas innovadoras. Revista Eureka sobre Enseñanza y Divulgación de las Ciencias, 16(3) 31023123. https://doi.org/10.25267/Rev_Eureka_ensen_divulg_cienc.2019.v16.i3.3102

Martín, R. D. y Tourón, J. (2017). El enfoque flipped learning en estudios de magisterio: percepción de los alumnos. RIED. Revista Iberoamericana de Educación a Distancia, 20(2), 187-211. https://doi.org/10.5944/ried.20.2.17704

Mengual Andrés, S., López Belmonte, J., Fuentes Cabrera, A., y Pozo Sánchez, S. (2020). Modelo estructural de factores extrínsecos influyentes en el flipped learning. Educación XX1. 23(1), 75-101. https://doi.org/10.5944/educxx1.23840

Miller, A. (February 24, 2012). Five Best Practices for the Flipped Classroom. Edutopia. Posted online. http://www.edutopia.org/blog/flipped-classroom-best-practicesandrew-miller

Nielsen, L. (2012). Five reasons I'm not flipping over the flipped classroom. Technology $\mathcal{E}$ Learning, 32(10), 10-46.

Prieto Martín, A. (2017). Flipped Learning: aplicar el modelo de aprendizaje inverso. Narcea Ediciones. 
Salinas Martínez, N. P. y Quintero Rodríguez, E. (2018). A hybrid and flipped version of an introductory mathematics course for higher education. Journal of Education for Teaching, 44(1), 112-117. https://doi.org/10.1080/02607476.2018.1422616

Sola Martínez, T., Aznar Díaz, I., Romero Rodríguez, J. M. y Rodríguez-García, A. M. (2019). Eficacia del método Flipped Classroom en la Universidad: Meta-análisis de la producción científica de impacto. REICE. Revista Iberoamericana sobre Calidad, Eficacia y Cambio en Educación, 17(1), 25-38. https://doi.org/10.15366/reice2019.17.1.002

Tourón, J., y Santiago, R. (2015). El modelo Flipped Learning y el desarrollo del talento en la escuela: Flipped Learning model and the development of talent at school. Revista de Educación, 368, 196-231. https://doi.org/10.4438/1988-592X-RE-2015-368-288

Vázquez-Alonso, Á. y Manassero-Mas, M. A. (2015). Hacia una formación inicial del profesorado de ciencias basada en la investigación. Revista Española de Pedagogía, (261), 343-363.

Yilmaz, R. M. y Baydas, O. (2017). An examination of undergraduates' metacognitive strategies in pre-class asynchronous activity in a flipped classroom. Educational Technology Research and Development, 65(6), 1547-1567. https://doi.org/10.1007/s11423017-9534-1

Zaunuddin, Z. y Halili, S.H. (2016). Flipped classroom research and trends from different fields of study. The international review of research in open and distributed learning, 17(3), 313-340. https://doi.org/10.19173/irrodl.v17i3.2274

Fecha de recepción: 25 de junio de 2020.

Fecha de revisión: 7 de julio de 2020.

Fecha de aceptación: 12 de noviembre de 2020. 